\title{
EVOCACIONES EN TORNO A LOS NOMBRES DE SOSIA Y TRISTAN
}

\section{Fernando Cantalapiedra Jaén}

Al llegar al auto trezeno, nadie puede negar una cierta extrañeza ante la súbita aparición de los dos nuevos criados, Sosia y Tristán. El hecho de que Calisto, al despertar, llame a éste último, desconocido hasta ese momento, causa también cierto desconcierto; se hubiera esperado en ese instante, como mínimo, la introducción de Sosia, el criado ausente en el acto II, y del que Pármeno informa "que no está Sosia en casa.» Aunque es muy posible que la citada frase esté también interpolada, entre otras razones, porque es absurdo pensar que un personaje inexistente pueda estar ausente. Cuando Rojas interpoló la citada frase tenía ya redactada la introducción de Sosia en el acto XIII, en el que Sosia sí estaba ausente de la casa de Calisto, dado que estaba en la plaza presenciando la ejecución de sus predecesores. La citada frase se produce además en el contexto del caballo, y el papel temático de «mozo de espuelas» de Sosia se desarrolla en el acto XIII. 
Partiendo de este papel temático, se ha pretendido establecer una relación jerárquica entre los criados de Calisto, olvidando que dicho papel lo asumen, en la Celestina primitiva, tanto Pármeno como Sempronio:

Sempronio. - Aqui estoy, señor, curando destos
cavallos. 1. 8
Calisto. - ¿Viene esse cavallo? ¿Qué hazes, Pármeno?
II. 27.1

Es posible que Sempronio esté mintiendo, pero para poder mentir es necesario hacerlo desde la óptica de lo posible: Dicho de otra manera, para que Sempronio pueda mentir es necesario que la tarea de cuidar de los caballos le esté encomendada. El que está ausente es Sempronio, quien por orden de su señor está acompañando a Celestina. Por tanto, la frase de Pármeno más que injerida está modificada, y es posible que el Anónimo hubiese escrito: que no está Sempronio en casa. En cuyo caso la frase anterior de Pármeno, II. 26, está modificada o injerida en parte o totalmente; y en particular: " $i M o c ̧ o s !$ ¿No ay moço en casa? ...moço de espuelas.» Esta frase sirve únicamente para preparar la introducción de los nuevos criados. En la versión del autor Anónimo, el proceso dialéctico con el que Calisto pretende conseguir la ayuda de sus criados se inicia y se concluye con la relación criado/caballo, en tanto que figura de la pasión y la lascivia. Tanto Sempronio como Pármeno participan en la preparación de la «montura» de Calisto, el primero «cuidando,» el segundo 'ensillando'; los demás moços ni existen ni participan en la seducción.

En los doce primeros actos, el papel temático «cuidador de caballos» aparece de forma implicita en el discurso, mientras que en el caso de Sosia aparece definido de forma explícita. Por tanto, la especialización temática de los criados empieza a señalarse en el acto XIII, y se confirma plenamente en el Tratado.

He señalado en otro lugar que la escena primera de la huerta está interpolada y es obra de Rojas. Además, Sempronio está cuidando de

1 Citamos siempre la edición crítica de Miguel Marciales, siguiendo su sistema de división del texto, "Tragicomedia de Calisto y Melibea, F. de Rojas.» Urbana/Chicago: University of Illinois Press, 1985, vol. II. 
los caballos y del girifalte; y sin éstos ningún caballero se va de caza, como lo desmuestran los propios grabados. LC se inicia en I. 8, estando Calisto en su casa, y la primera salida de éste se produce cuando sus criados terminan de prepararle el caballo, al final del segundo acto. En su segunda salida se mencionan las figuras del caballo y del asno: "Los cavallos de Febo", VIII. 43, y "lo convirtió en asno», VIII. 48.

Por otro lado, Celestina enseña a Pármeno que el verdadero placer está en la comunicación del deleite y no sólo en el acto en sí. Teoria que aplica el mozo al narrar a Sempronio su noche de amor con Areúsa. Sin embargo, Calisto, al despertar en el acto XIII, no habla de comunicar sino de confirmar su gozo, de saber si su cita con Melibea fue un simple sueño o si fue realidad, recordando en realidad su frase del acto XI. 15.

Nos llama también la atención la utilización del diminutivo-iTristanico!-, ya que es totalmente extraño a las interrelaciones anteriores entre Calisto y sus criados. La presencia de Tristán delante de la puerta de la casa es insólita, dado que con anterioridad esta presencia estaba reservada a los personajes femeninos y se relacionaba con el código social de la espera ante la puerta antes de traspasar su umbral. El final del acto XII es caótico y problemático; el salto nada tiene que ver con la caída, y desde luego no se elabora ninguna estrategia narrativa que justifique la transformación del salto en caída, accidente, detención, enjuiciamiento, pregón y ejecución pública, todo ello en el transcurso de unas horas.

En todo caso, no es nuestro propósito realizar un estudio, en estas líneas, sobre los nuevos criados, ni tan siquiera el de analizar las situaciones extrañas a las que acabamos de referirnos someramente. Nuestro objectivo se limita a una simple lectura evocativa de los nombres de los nuevos criados. Nuestra hipótesis de partida es que los nombres propios de los nuevos criados condicionan en buena medida los cuatro últimos actos de la Comedia. El nombre de Sosia, ( $\sim$ parecidos), establece un paralelismo entre los nuevos y los viejos criados. Por otro lado, si Sosia comunica la muerte de Pármeno y Sempronio, el nombre de Tristán anuncia la muerte de Calisto y Melibea. 


\section{Tristán}

Se sabe que el nombre de Tristán tiene su origen en el ciclo de la literatura bretona: Bonilla y San Martín y Menéndez Pelayo propusieron la influencia de la leyenda de Tristán en Celestina, pero Castro Guisasola se encargó de desmentir dicha influencia. Sin entrar en este tipo de debate, es conveniente recordar algunos hechos literarios en torno al Tristán:

«Una de las más antiguas [referencias] es debida al catalán Guirau de Cabrera, quien, con toda seguridad antes del año 1160, reprochaba a su juglar que nada supiera

$$
\begin{aligned}
& \text { ni del vil nan } \\
& \text { ni del Tristan } \\
& \text { c' amava Yceut a lairon. }
\end{aligned}
$$

(ni del vil enano, ni de Tristán que amaba a Iseo a hurtadillas). (...) $\mathrm{Y}$ pocos años antes el trovador Cercamon había escrito en una canción que tenía «lo cor tristan», o sea el corazón entristecido, haciendo juego de palabras con el nombre del famoso enamorado. (...)

El romancero castellano acoge la figura de don Tristán, y uno de los romances sobre este personaje era una de las canciones más apreciadas por las damas de Isabel la Católica."

Recordemos también que el cuento de Tristán, en sus distintas versiones, se extendió rápidamente por todo el mundo occidental y que ya en el siglo XIV existian traducciones castellanas y catalanas. Al repasar los libros de Fernando de Rojas, Stephen Gilman nos recuerda que:

«entre cerca de sesenta libros no profesionales, quedan configurados no menos de diez libros de ese género (de caballerias) y una de las varias versiones de la historia de Tristán e Isolda»;

2 M. de Riquer y J. M. Valverde, Historia de la literatura universal, III (Barcelona: Planeta, 1984), págs. 120 y 125. 
$y$ añade en nota el insigne profesor:

«El 'Don Tristán' consignado en 1546 podría haber sido el libro del esforçado cavallero don Tristán de leonís (Valladolid, 1501, y Sevilla, 1528 y 1533); la crónica de don Tristán de Leonis en español (Sevilla, 1520) o la Crónica nuebamente enmendada y añadida del buen cavallero don Tristán de Leonis (Sevilla, 1534). ${ }^{3}$

Sin entrar en detalles de qué versión, o versiones, pudo tener a mano Fernando de Rojas, sí puede decirse que el tema de Tristán interesaba a la sociedad de aquella época, y que también le interesaba a este autor. Por otro lado, tampoco pretendemos estudiar las posibles fuentes textuales de Celestina en la leyenda de Tristán, nos limitaremos a ciertas generalidades comunes a ellos.

El Tristán bretón recibió este nombre para señalar que a causa de su nacimiento murió su madre, Blancaflor, y significó sencillamente el «triste.» Es conveniente recordar algunas características de la leyenda: Tristán, en su viaje a Irlanda para evitar ser reconocido, cambia de nombre, trastocando las silabas, y se hace llamar «Tantris.» Tras sus aventuras amorosas con la rubia Iseo, experta, como Celestina, en medicina y magia, y con Iseo, la de las blancas manos, concluye la leyenda con la trágica muerte de Tristán e Iseo la rubia, muertes causadas por la otra Iseo.

Resulta interesante observar los paralelismos narrativos evocados con la simple introducción del nombre de Tristán en el acto XIII de Celestina:

a) También Tristán «nace» en Celestina tras la muerte de la «madre» Celestina, y de los criados Pármeno y Sempronio.

b) La aparición de Tristán, el triste, evoca a su vez el nuevo sesgo que toma Celestina: «que no se avia de llamar comedia, pues acabava en tristeza» (P 25).

c) El cambio de nombre de Tristán por Tantris, recuerda que estamos ante dos nuevos nombres en lo que debiera ser

3 S. Gilman, La España de Fernando de Rojas, (Madrid: Taurus, 1978), pág. 424. 
el epílogo de la obra, y ante un cambio de personalidad y de personaje.

d) El propio nombre evoca, y más aún en aquella época, el final trágico de Tristán e Iseo, y sirve de preparación narrativa a la muerte de Calisto y Melibea.

Parece obvio que Fernando de Rojas seleccionó con sumo cuidado el nombre de Tristán, y que lo utilizó como una catáfora, que tiene la virtud de funcionar como un nombre resumen de la acción narrativa que se desarrollará tras su entrada en la obra. Es posible que dicho nombre tenga a su vez la función de avisar al lector de otra cosa; por ejemplo, del cambio de autor. Esta suposición implica una lectura hermenéutica de los apartados anteriores:

$\mathbf{a}^{1}$ ) Tristán «nace» en Celestina tras el final («muerte») del manuscrito del autor anónimo. Operando desde el binomio complementario: /maternidad $\rightarrow$ paternidad literaria/.

$\left.b^{1}\right)$ El «Triste» nace causando el final («muerte») de la comedia anónima, dando paso a la «vida» de la tragedia de Rojas.

$\left.c^{1}\right)$ El cambio de nombre, Tristán $\rightarrow$ Tantris, para no ser reconocido, pudiera muy bien connotar el cambio de autores y las octavas acrósticas en donde Fernando de Rojas, o Proaza, encubre-descubre su nombre y condición.

$\left.d^{1}\right)$ La leyenda de Tristán es la historia de un amor trágico y de dos mujeres con el mismo nombre. Partiendo de la constatación de que la rubia Iseo asume algunos de los papeles temáticos de Celestina, experta en medicina y magia, un paralelismo literario resulta fácil de establecer:

/La rubia Iseo: $L C$ anónima:: Iseo la blanca: $L C$ de Rojas/

Es decir, las dos Iseos connotan las dos Celestinas. Recuérdese que si la primera Iseo curó y salvó la vida de Tristán, la segunda causó la muerte de los dos amantes; lo mismo sucede en nuestra obra, en donde Celestina, anónima, cura y une a los dos enamorados, y Rọas, segunda Iseo, provoca la trágica muerte de Calisto y Melibea. En este contexto evocativo, toma nuevo sentido la frase de Melibea: 
«Subamos, señor, al açotea alta, por que desde allí goze de la deleitosa vista de los navíos, por ventura afloxará algo mi congoxa.» Esto trae a la memoria el regreso del navio de la rubia Iseo, que retorna para salvar de nuevo la vida de Tristán, que se muere de amor por ella, y la traición de la otra que le miente al comunicarle desde la ventana de la torre que las velas del barco son negras, y que por tanto la rubia Iseo no vendrá a su lecho de muerte.

Los paralelismos literarios entre la leyenda de Tristán y la Celestina pueden resumirse de un modo más gráfico:

\begin{tabular}{|c|c|c|}
\hline CURACION-VIDA & - VS - & NO CURACION-MUERTE \\
\hline Anónimo : $\underline{1}^{\underline{a}}$ Iseo & & Rojas $: \underline{2^{2}}$ Iseo \\
\hline Calisto`Melibea Tristán & - VS - & Calisto $_{\mathbf{v}}$ Melibea Tristán $\mathbf{v}_{\mathbf{V}}$ Iseo \\
\hline UNION $\approx$ VELA BLANCA & - Vs - & EPARACION $\approx$ VELA NEGRA \\
\hline
\end{tabular}

\section{Sosia}

El nombre de Sosia merece unas consideraciones previas:

a) Es un personaje de la comedia Anfitrión de Plauto;

b) Es asimismo un personaje terenciano y aparece en $L a$ Andriana y en La Suegra;

c) Persona que tiene parecido con otra hasta el punto de poder ser confundida con ella."

Si se toma como punto de partida los datos de los apartados a) y b), se deduce que el mismo nombre es utilizado por dos autores diferentes en tres obras dramáticas. Es decir, el personaje de Sosia es utilizado una vez por Plauto y dos veces por Terencio; por tanto, en lectura hermenéutica, el contacto entre los dos autores latinos connota la cifra doce, que puede interpretarse como el momento del encuentro de Rojas con el manuscrito anónimo. Siguiendo con esta evocación libre y aparentemente absurda, pero apoyada en hechos reales,

4 Diccionario de la Lengua española, Madrid: R.A.E., Vigésima ed., 1984. 
tenemos que Sosia sólo aparece en el primer acto de La Andriana, en el tercero de La Suegra y en cuatro actos del Anfitrión. También sabemos que Terencio es un autor posterior a Plauto. La reorganización hermenéutica de esta serie de datos puede presentarse como sigue:

APARICIONES DE SOSIA EN TERENCIO Y PLAUTO

\begin{tabular}{|c|c|c|c|}
\hline Comedias: & & ANDRIANA / SUEGRA & ANFITRION \\
\hline Acto(s) & & / III & en cuatro \\
\hline Connotación & & XIII & XIII, XIV, XV, XVI \\
\hline TERENCIO & : & ROJAS:: POSTERIORIDAD & ACTOS DE ROJAS \\
\hline
\end{tabular}

Esta asimilación de Rojas con los autores latinos puede parecer sorprendente, pero debe recordarse que tanto Plauto como Terencio se inspiraron para su creación literaria en la obra de Menandro. Ahora bien, de este autor griego sólo se conocían fragmentos de sus obras hasta el siglo XIX. ${ }^{5}$ De la reorganización hermenéutica de los datos anteriores con los últimos, se desprenden las equivalencias siguientes:

\begin{tabular}{|c|c|c|c|c|}
\hline Temporalidad: & ANTERIORIDAD & . & & POSTERIORIDAD \\
\hline autores & Anónimo & Menandro & Rojas & Plauto/Terencio \\
\hline obra & inacabada & fragmentos & acabar & : completa(r) \\
\hline clasicismo & ANONIMO & GRIEGOS & $::$ ROJAS & : LATINOS \\
\hline
\end{tabular}

Es decir, el autor anónimo es a Menandro lo que Rojas es a Plauto y Terencio, y así sucesivamente. Claro que se puede objetar que todo esto es un simple juego del que escribe, y se acepta, pero seria olvidar que en cierta medida todo ello ya se rememora en las octavas

5 «Como es sabido, por vez primera algunos fragmentos de Menandro (procedentes de las citas diseminadas en obras antiguas) en Paris por G. Morel, 1533 con el título «Ex Comoediis Menandri quae supersunt.» F. Castro Guisasola, Observaciones sobre las fuentes literarias de La Celestina (Madrid, C.S.I.C., 1973, reimpresión), pág. 19. 
acrósticas, en sus dos variantes principales:

«Jamás yo no vi una Terenciana/jamás yo no vide en lengua romana»

«En lengua común vulgar castellana/en tusca, ni griega, ni en castellana» (OA. 9).

Mientras que en la primera variante se introduce la oposición /Terenciana vs. castellana/, en la segunda se emplea una doble oposición: /(griega vs castellana) vs (romana vs castellana). De tal modo que si la «lengua romana» es el equivalente de "Terenciana,» la lengua «tusca» aparece como el equivalente de la obra de Plauto el toscano. Ahora bien, si Plauto y Terencio se inspiraron de los autores griegos y en particular de Menandro, en las octavas acrósticas se da a entender que Rojas se inspiró a su vez del autor anónimo. Toda esta serie de paralelismos queda reflejada en el siguiente diagrama:

POSTERIORIDAD

Plauto/Terencio

Rojas $\rightarrow$

SE INSPIRA(N)
ANTERIORIDAD

Griegos/Menandro

Siguiendo en esta línea de lectura evocativa, conviene recordar algunos datos literarios harto conocidos, pero a los que no se ha concedido importancia alguna en lo que se refiere a Celestina. El autor del prólogo a La Andriana, es posible que fuera el propio Terencio, aunque hable en tercera persona quien nos recuerda que:

«Menandro compuso La Andriana y La Perintia. Quien la una de ellas conociere bien, conocerá las dos, según ambas son de argumento semejante, aunque por el diálogo y estilos diferentes. Todo lo que de la Perintia cuadraba para La Andriana Terencio confiesa haberlo trasladado, sirviéndose de ello cual si fuese de su propia invención. $Y$ esto es lo que sus enemigos le censuran. Porque dicen que no es bien hacer de varias una sola fábula.» ${ }^{6}$

6 Publio Terencio Afer, col. Austral (Madrid: Espasa-Calpe, 1947), pág. 9. 
Los paralelismos con el problema de la autoría de la Celestina parecen evidentes: dos obras en una, dos autores y el mismo título. Lo cual trasladado al diagrama anterior da como resultado:

$\begin{array}{lcl} & \text { ANDRIANA } \approx \text { LA CELESTINA } \\ \text { ANDRIANA }: & \frac{\text { Terencio }}{\text { Andriana }} \rightarrow & \frac{\text { Menandro }}{\text { Andriana-Perintia }} \\ \text { LA CELESTINA: } & \frac{\text { Rojas } \rightarrow}{\text { Celestina } .} & \frac{\text { Anónimo }}{\text { Celestina }}\end{array}$

Lo dicho en el prólogo a La Andriana recuerda un poco lo que se nos dice en el material liminar de Celestina. En este sentido, merece la pena también citar un párrafo del prólogo al Anfitrión de Plauto:

«Mercurio.- Haré que esta representación se convierta de una tragedia en una comedia. De tal manera que se conservará todos sus mismos versos. (...) $Y$ haré que sea una cosa mezclada: una tragicomedia (...) esta noche ha sido prolongada, para hacer más largos sus placeres. Pero también conviene saber que hállase disfrazado como si fuera el propio Anfitrión.» ${ }^{7}$

Es posible negar toda posible interferencia de este pasaje con nuestra Celestina; sin embargo existen al menos cuatro elementos que merecen la pena ser retenidos:

a) La conservación de lo ya escrito $\approx$ lo que Rojas conserva del autor anónimo.

b) La prolongación del deleite $\approx$ la continuación de Rojas y el «Tratado de Centurio» en particular.

c) La mezcla de placer y de tragedia $\approx$ la tragicomedia de Rojas

d) El personaje disfrazado $\approx$ el autor encubierto.

No obstante, la crítica ha retenido el único elemento no pertinente; es decir, la oposición binaria: / (divinidades vs humanos) vs (comedia vs

7 Plauto, «Comedias," versión de J. B. Xuriguera (col. Obras maestras, Barcelona, 1972), pp. 96-98. 
tragedia) /. Los cuatro elementos que hemos seleccionado como pertinentes se encuentran a su vez en el material liminar de Celestina:

«Otros an litigado sobre el nombre, diziendo que no se havía de llamar comedia, pues acavaba en tristeza, sino que se llamase tragedia. El primer autor quiso darle denominación del principio que fue plazer, y llamóla comedia (P 26). Yo, viendo estas discordias, entre estos estremos parti agora por medio la porfia, y llaméla tragicomedia (...) y hallé que querían que se alargasse en el proceso de su deleite destos amantes (P 25-26).

(...) quiso celar y encubrir su nombre, no me culpéis si, en el fin baxo que lo pongo, no espressare el mío (C. 9).»

Mientras que en El Anfitrión la tragedia se transforma en una comedia, en Celestina la comedia anónima da paso a la tragedia de Rojas, (Comedia = 12 primeros actos) vs (Tragedia $=$ cuatro últimos), $y$ ambas se transforman en tragicomedia respetando lo ya escrito y prolongando el deleite nocturno de los amantes. Finalmente, tanto Anfitrión como Rojas disfrazan su personalidad haciéndose pasar por otro. En todo caso, una cosa está bastante clara, el autor anónimo escribió una comedia, como se desprende de las propias palabras de Fernando de Rojas. Ahora bien ¿de cuántos actos? Lo que si sabemos con certeza es que la tragedia comienza hacia el final del duodécimo acto, lo que implica la imperiosa necesidad de crear nuevos personajes.

Volviendo ahora al nombre de Sosia, tomaremos como elemento de análisis el apartado c):

c) Persona que tiene parecido con otra hasta eì punto de poder ser confundida con ella.

El tema de los mellizos fue tratado por Menandro en La trasquilada, y posteriormente por Plauto en Los dos Menecmos. Merece la pena recordar un pasaje del prólogo de ésta última:

Cuando llegó la noticia a Siracusa y al abuelo de los niños, de que uno de sus nietos se habia perdido y el padre había muerto en Tarento, cambió el nombre del otro gemelo. Tanto quería al desaparecido, que puso su 
nombre al niño que quedaba (Sósicles), y desde entonces le llamaron Menecmo, que es como se llamaba su hermano. ${ }^{8}$

Estamos ante un ceremonial de la confusión, que implica personajes parecidos y cambios de nombres: /(Tristán $\longrightarrow$ Tantris) (Sósicles $\longrightarrow$ Menecmo)/. O lo que es literariamente equivalente, los nuevos criados de Celestina son parecidos, en las intenciones de Rojas, a los anteriores y ya desaparecidos, con la única diferencia del consabido cambio de nombres: Sosia y Tristán son los SOSIAS de Sempronio y Pármeno. $\quad \mathcal{O}$ es acaso una simple errata de imprenta la palabra SOSIAS, refiriendose al personaje, que aparece en el prólogo del acto XIII de las ediciones de Burgos 1499, de Toledo 1500, y de Sevilla 1501 ?

De lo señalado hasta el presente se infiere, primero, que la referencia literaria personaje/autor puede relacionarse con la confusión de personas parecidas. Es decir, Rojas pretende que se le confunda con el autor anónimo. Segundo, que los nuevos criados pueden confundirse con sus predecesores desaparecidos, al igual que en el caso de Menecmo. Tercero, que la muerte del padre de los dos niños puede interpretarse a su vez como la muerte del creador de Pármeno y Sempronio. Lo cierto en todo esto es que la historia de la autoría de Celestina tiene cierta semejanza con la comedia Anfitrión de Plauto, a la que su autor también llamó tragicomedia. Merece la pena citar el resumen de esta comedia hecho por Martin de Riquer:

«...Júpiter, enamorado de Alcmena, toma la forma de su esposo, Anfitrión, mientras éste defiende a la patria luchando contra el enemigo. Bajo la apariencia de Sosia, esclavo del auténtico Anfitrión, Mercurio consigue engañarlos. A su regreso, Anfitrión se irrita contra su mujer, y ambos maridos se acusan mutuamente de adulterio. Es designado árbitro de la disputa el piloto Blefarón, el cual se confiesa incapaz de distinguir cuál de los dos es el Anfitrión verdadero. Todo se aclara cuando Júpiter, desde el cielo, se

8 Plauto, Comedias, p. 208 (ver nota 7). 
confiesa ser el adúltero, y Alcmena da a luz dos gemelos.» ${ }^{9}$

En el marco de nuestra lectura evocativa y hermenéutica, se puede leer el pasaje citado estableciendo un paralelismo con el problema de la paternidad de Celestina: con la ayuda de Mercurio/Sosia, Júpiter/Rojas toma la forma de Anfitrión/Anónimo para conseguir los favores de Alcmena/Celestina, la cual da a luz gemelos; la doble paternidad implica que Alcmena tenga dos hijos y que la obra literaria conste de dos partes. Al mismo tiempo que se le sugiere al árbitro/lector su incapacidad para poder distinguir a los (pro)creadores y a lo creado por cada uno de ellos, se le sugiere que en todo caso en el cielo se sabrá toda la verdad.

Proaza, en la octava que cierra la obra, sigue haciendo referencia a los gemelos:

El carro febeo, después de aver dado

mil y quinientas bueltas en rueda

ambos entonces los hijos de Leda

a Febo en su casa tenién posentado (AP 6).

Miguel Marciales indica en nota:

Los hijos de Leda, los Gemelos, tenian el sol en su casa o signo, es decir, el sol estaba en Géminis, 11 de mayo al 12 de junio, en el calendario antes de la reforma gregoriana.

Es decir, la impresión del libro se terminó entre el 11 de mayo y el 12 de junio; no se especifica por tanto un dia preciso. Pero entonces ¿por qué las siguientes ediciones cambian la fecha del año y no la del mes? ¿0 es qué todas se editaron en el mismo mes? ¿se trata en realidad de un problema de métrica y de rima? Es posible que sea eso, pero también conviene recordar que el material liminar sufrió bastantes cambios y modificaciones. En todo caso, la denotación de la octava final de Celestina conlleva una doble referencia a la cif ra doce: el doce de junio y los doce signos del zodiaco.

Una lectura mitológica del último pasaje citado nos conduce hacia una metáfora similar a las anteriores. Febo es el epiteto del dios

$\overline{9}$ Obra citada (n. 2), vol. I, pág. 268. 
Apolo, quien provocó los partos dobles en las vacas del rey Admeto. Leda es la madre de las dos parejas de gemelos, Helena y Pólux, Cástor y Clistemestra, fecundados la misma noche por dos dioses diferentes, Zeus y Tindáreo. ${ }^{10}$

Puede proponerse otra lectura isotópica desde la optica del zodíaco, ya que la constelación de Géminis se caracteriza por sus dos principales estrellas, Cástor y Pólux, muy brillantes y cercanas. ${ }^{11}$ Lo que puede interpretarse como: el sol/Celestina está (escrita) entre Cástor y Pólux/Anónimo y Rojas, dos estrellas/autores muy cercanos y brillantes.

Hasta aqui nuestra lectura libre y evocativa en torno a los nombres de los nuevos criados; pero debemos recordar que, a pesar de las libertades tomadas, hemos permanecido atados al cordón del texto de la Comedia, puesto que los autores clásicos que hemos utilizado están citados en el material liminar. Baste una última cita:

No debuxó la cómica mano de Nevio ni Plauto, varones prudentes, tan bien los engaños de falsos sirvientes y malas mugeres, en metro romano.

Cratino y Menandro y Manes anciano esta materia supieron apenas pintar en estilo primero de Atenas, como este poeta en su castellano. (AP 3)

«En estilo primero de Atenas» escribió Cratino, según la tradición, veintiuna comedias, con las cuales obtuvo nueve premios en las Dionisiacas y en las Leneas. ${ }^{12}$ Es considerado como el primer poeta que dio a la comedia una verdadera dignidad literaria. Lo mismo puede decirse de Celestina y de su autor anónimo.

En conclusión, los nombres de Sosia y de Tristán evocan toda una serie de lecturas posibles, incluso antes de desarrollarse como personajes, tendentes a manifestar la ruptura existente entre los doce primeros actos, en los que ellos están ausentes, y el resto de la

10 C. Falcón y otros, Diccionario de la Mitología Clásica. Madrid: Alianza, 1983. 2 vols.

11 Dictionnaire Larousse. Paris, 1977.

12 Diccionario de Autores. Barcelona, Montaner y Simón, 1973. 
Tragicomedia. De la mano de Rojas, los nuevos criados, los Sosias, nacen con la muerte anunciando otra muerte;. Sosia germina como testigo y comunicante de las ejecuciones de Pármeno y de Sempronio; Tristán, que con su nombre evoca ya toda la tragedia, cierra su ciclo escénico anunciando la muerte de Calisto, y la Gran Adición completa el ciclo reservándole el papel de único testigo presencial del trágico accidente. Los dos criados llegan a Celestina con la muerte y se van con la muerte, cantando ante el cadáver de su señor. Esta estructuración de la substitución, evocación, muerte y comunicación queda reflejada en el gráfico siguiente:

MUERTE

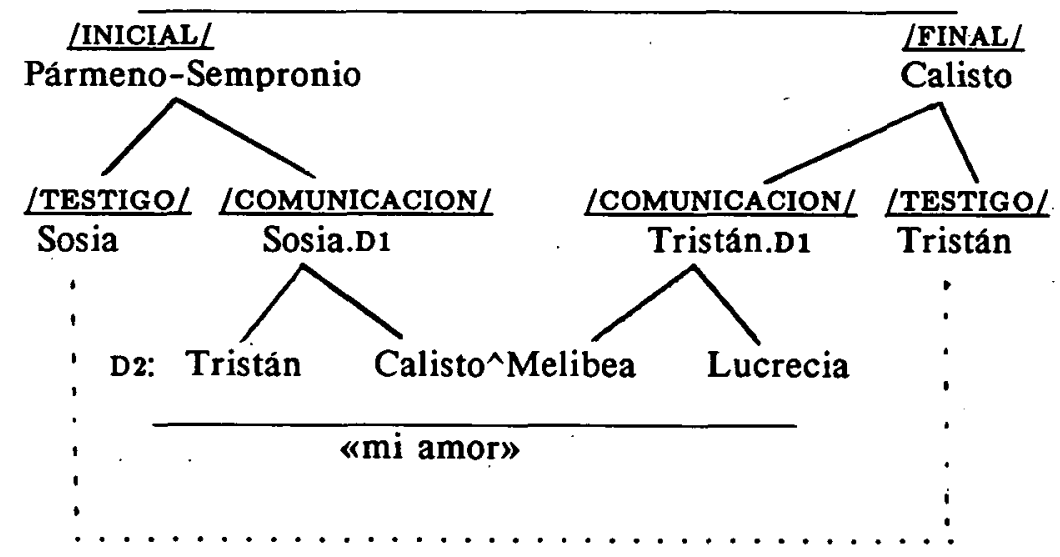

N.B. . . . . = La gran adicion. = Los cuatro últimos actos de la Comedia.

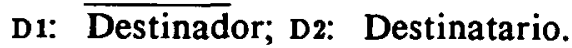




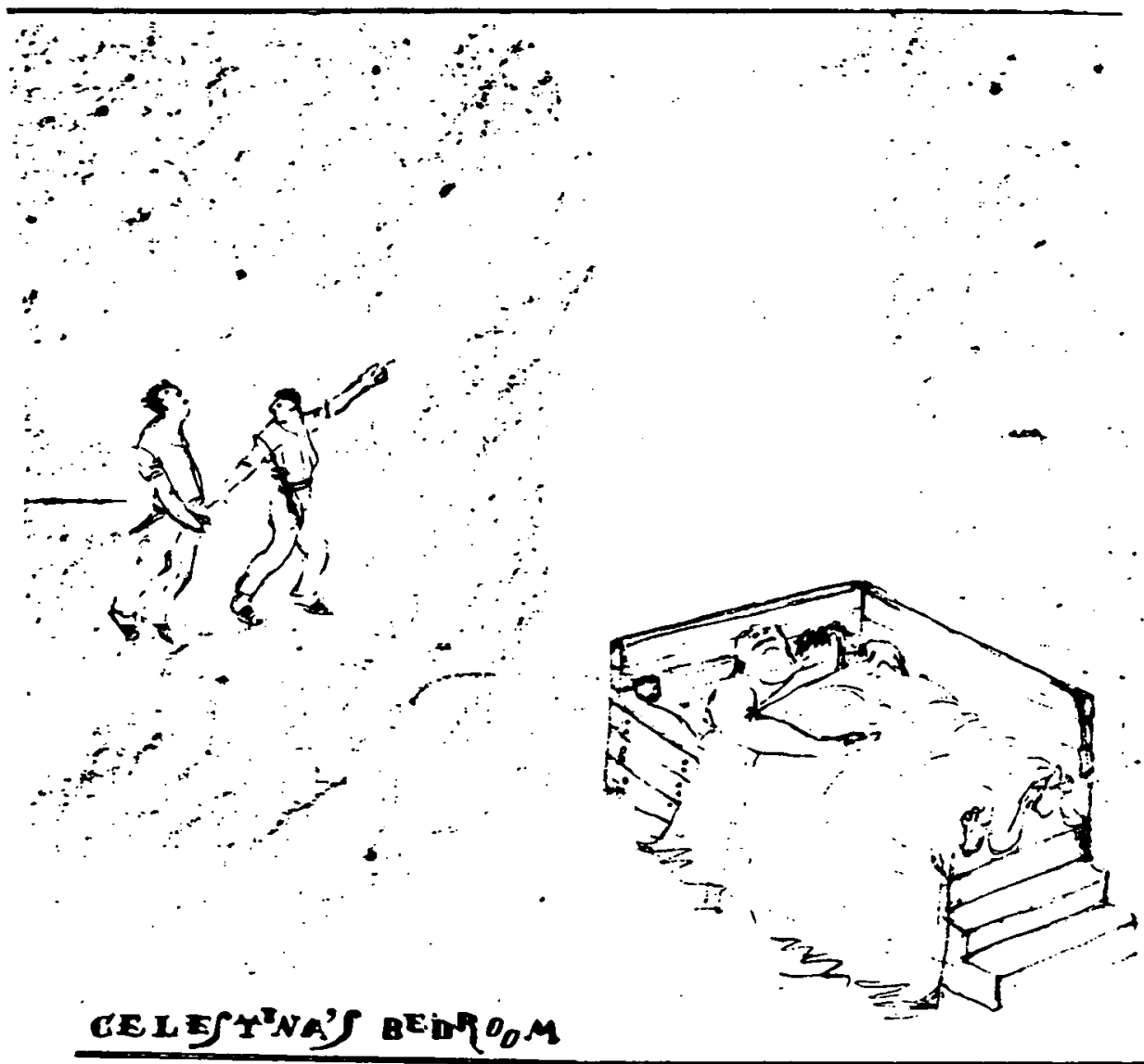

ACTO XII"

Pamela HOWARD 